\title{
MODELING THE 3-D FLOW AROUND A CYLINDER USING THE SAS HYBRID MODEL
}

\author{
STAMOU A.I. ${ }^{*}$ \\ PAPADONIKOLAKI G.
}

\author{
Department of Water Resources and Environmental Engineering \\ School of Civil Engineering, National Technical University of Athens \\ Heroon Polytechniou 5, 15780 Athens, Greece
}

Received: 29/05/2014

Accepted: $12 / 11 / 2014$

Available online: $20 / 11 / 2014$ *to whom all correspondence should be addressed: e-mail: stamou@central.ntua.gr

\section{ABSTRACT}

Three-dimensional calculations were performed to simulate the flow around a cylindrical vegetation element using the Scale Adaptive Simulation (SAS) model; commonly, this is the first step of the modeling of the flow through multiple vegetation elements. SAS solves the Reynolds Averaged NavierStokes equations in stable flow regions, while in regions with unstable flow it goes unsteady producing a resolved turbulent spectrum after reducing eddy viscosity according to the locally resolved vortex size represented by the von Karman length scale. A finite volume numerical code was used for the spatial discretisation of the rectangular computational domain with stream-wise, cross-flow and vertical dimensions equal to 30D, 11D and 1D, respectively, which was resolved with unstructured grids. Calculations were compared with experiments and Large Eddy Simulations (LES). Predicted overall flow parameters and mean flow velocities exhibited a very satisfactory agreement with experiments and LES, while the agreement of predicted turbulent stresses was satisfactory. Calculations showed that SAS is an efficient and relatively fast turbulence modeling approach, especially in relevant practical problems, in which the very high accuracy that can be achieved by LES at the expense of large computational times is not required.

Keywords: Cylinder, Vortex shedding, Turbulent wake, Hybrid models, Scale adaptive simulation (SAS)

\section{Introduction}

The flow around a cylinder, which is frequently encountered in many practical engineering problems, including the flow in flood plains, has been extensively studied over the last five decades. The nature of this flow that combines a simple geometry with complicated flow phenomena, such as flow separation and vortex shedding, is determined by the Reynolds number $\left(R_{D}\right)$ based on cylinder diameter (D) and free-stream velocity $\left(U_{\infty}\right)$, which identifies several distinct flow regimes (Roshko, 1954; Kravchenko and Moin, 2000). At Reynolds numbers up to approximately $\operatorname{Re}_{D} \approx 40$ the entire flow is laminar, steady and symmetrical with a pair of symmetric counter-rotating vortices forming behind the cylinder (at $\left.\operatorname{Re}_{D}>5\right)$; in the stable range $R_{D} \approx 40-150$ the flow is entirely laminar and a regular and stable vortex shedding (Karman vortex street) is observed (Roshko, 1954). Within the range $\mathrm{Re}_{\mathrm{D}} \approx 190-260$ the flow is characterized by dominant vertical scales having wavelength of approximately four cylinder diameters. At $R e_{D} \approx 260$, the flow transits to finer scale three dimensionality with vertical characteristic length equal to around one cylinder diameter. With increasing Reynolds number above 300 the flow is characterized as irregular (Roshko, 1954) exhibiting a Strouhal number (St) that is relatively independent of Reynolds number and the three-dimensional (3-D) cylinder wake becomes more chaotic; the latter is believed to lead to the reduction in the base suction coefficient defined as the negative of the base pressure 
coefficient $\left(-\bar{C}_{p b}\right)$. The shear layers separating from the cylinder become unstable at a critical Reynolds number that varies from 300 to 3000 (Prasad and Williamson, 1996). At Reynolds numbers between $2 \times 10^{5}$ and $3.5 \times 10^{6}$ a dramatic decrease in the base suction coefficient occurs that is associated with a complicated flow regime with laminar separation, transition to turbulence, reattachment, and another separation further downstream along the cylinder surface. At Reynolds numbers beyond $3.5 \times 10^{6}$, the boundary layer on the cylinder becomes turbulent before separation and the flow is characterized by a lower base suction coefficient and lower drag due to delayed separation of the turbulent boundary layer.

Table 1. Numerical details and overall flow parameters

\begin{tabular}{|c|c|c|c|c|c|c|c|c|c|c|c|}
\hline Researchers & $L_{x} / D$ & $\mathrm{~L}_{y} / \mathrm{D}$ & $\mathrm{L}_{2} / \mathrm{D}$ & $\begin{array}{l}N_{\text {total }} \\
\left(\times 10^{6}\right)\end{array}$ & $\mathrm{n}_{\mathrm{z}}$ & $U_{\infty} d t / D$ & $\mathrm{~N}_{\mathrm{T}}$ & $-\bar{C}_{p b}$ & $\overline{\mathrm{C}}_{\mathrm{D}}$ & St & Lrec/D \\
\hline Experiments & - & - & - & - & - & - & - & $\begin{array}{c}0.83 \\
-0.93\end{array}$ & $\begin{array}{c}0.94 \\
-1.04\end{array}$ & $\begin{array}{c}0.205 \\
- \\
0.215 \\
\end{array}$ & $\begin{array}{c}1.13 \\
-1.53\end{array}$ \\
\hline $\begin{array}{c}\text { Young and OOi } \\
(2007), 2 \text { D-URANS }\end{array}$ & 40 & 30 & 0 & 0.09 & 1 & 0.050 & 30 & 1.96 & 1.59 & 0.235 & - \\
\hline $\begin{array}{c}\text { Young and OOi } \\
\text { (2007), } \\
\text { 3D-URANS }\end{array}$ & 40 & 30 & $\pi$ & 1.44 & 16 & 0.050 & 30 & 1.42 & 1.32 & 0.223 & - \\
\hline $\begin{array}{c}\text { Young and OOi } \\
(2007), \text { LES }\end{array}$ & 40 & 30 & $\pi$ & 0.36 & 4 & 0.004 & 30 & 1.86 & 1.55 & 0.217 & - \\
\hline $\begin{array}{c}\text { Young and OOi } \\
(2007), \text { LES }\end{array}$ & 40 & 30 & $\pi$ & 1.40 & 16 & 0.004 & 30 & 1.36 & 1.25 & 0.196 & - \\
\hline $\begin{array}{c}\text { Young and OOi } \\
(2007), \text { LES }\end{array}$ & 40 & 30 & $\pi$ & 2.90 & 32 & 0.004 & 30 & 0.913 & 1.04 & 0.212 & - \\
\hline $\begin{array}{c}\text { Young and OOi } \\
(2007), \text { LES }\end{array}$ & 40 & 30 & $\pi$ & 4.30 & 48 & 0.004 & 30 & 0.908 & 1.03 & 0.212 & - \\
\hline $\begin{array}{l}\text { Kravchenko and } \\
\text { Moin (2000), LES }\end{array}$ & 36 & 50 & $\pi / 2$ & - & 4 & 0.005 & 35 & 1.21 & 1.36 & 0.190 & 1.04 \\
\hline $\begin{array}{l}\text { Kravchenko and } \\
\text { Moin (2000), LES }\end{array}$ & 36 & 50 & $\pi / 2$ & - & 24 & 0.005 & 35 & 0.97 & 1.07 & 0.212 & 1.30 \\
\hline $\begin{array}{l}\text { Kravchenko and } \\
\text { Moin (2000), LES }\end{array}$ & 36 & 50 & $\pi$ & - & 8 & 0.005 & 35 & 1.23 & 1.38 & 0.193 & 1.00 \\
\hline $\begin{array}{l}\text { Kravchenko and } \\
\text { Moin (2000), LES }\end{array}$ & 36 & 50 & $\pi$ & 0.51 & 48 & 0.005 & 35 & 0.98 & 1.07 & 0.206 & 1.04 \\
\hline $\begin{array}{l}\text { Kravchenko and } \\
\text { Moin (2000), LES }\end{array}$ & 36 & 50 & $\pi$ & 2.41 & 48 & 0.005 & 35 & 0.93 & 1.04 & 0.210 & 1.35 \\
\hline Ma et al. (2000), DNS & 40 & 18 & $2 \pi$ & & 128 & - & $>33$ & 0.96 & - & 0.203 & 1.12 \\
\hline Ma et al. (2000), DNS & 40 & 18 & $\pi$ & & 64 & - & $>33$ & 0.84 & - & 0.219 & 1.59 \\
\hline Ma et al. (2000), DNS & 40 & 18 & $1.5 \pi$ & & 32 & - & $>33$ & 1.04 & - & 0.206 & 1.00 \\
\hline $\begin{array}{c}\text { Present work,3D- } \\
\text { URANS }\end{array}$ & 30 & 11 & 1 & 1.47 & 10 & 0.002 & 30 & 0.87 & 0.88 & 0.222 & 1.03 \\
\hline Present work, SAS & 30 & 11 & 1 & 0.38 & 5 & 0.002 & 30 & 0.96 & 0.94 & 0.221 & 1.05 \\
\hline Present work, SAS & 30 & 11 & 1 & 1.47 & 10 & 0.002 & 10 & 0.94 & 0.90 & 0.229 & 1.10 \\
\hline Present work, SAS & 30 & 11 & 1 & 1.47 & 10 & 0.002 & 20 & 0.93 & 0.90 & 0.228 & 1.08 \\
\hline Present work, SAS & 30 & 11 & 1 & 1.47 & 10 & 0.002 & 30 & 0.93 & 0.90 & 0.228 & 1.08 \\
\hline Present work, SAS & 30 & 11 & 1 & 2.51 & 10 & 0.002 & 30 & 0.93 & 0.90 & 0.228 & 1.08 \\
\hline
\end{tabular}

In the present work we investigate the case of the Reynolds number $\operatorname{Re}_{\mathrm{D}}=3900$ for three reasons. Firstly, this case is within the range of values found in the modeling of vegetation elements (Lopez and Garcia, 2001; Stamou et al., 2012). Secondly, a comprehensive range of experimental data is available at this Reynolds number that can be used for model assessment (Cardell, 1993; Norberg, 1994; Lourenco and 
Shih, 1993; Ong and Wallace, 1996), which include overall flow parameters, such as $-\bar{C}_{p b}$, the drag coefficient $\left(C_{D}\right)$, St and the recirculation length $\left(L_{\text {rec }}\right)$, mean and turbulent flow characteristics; the values of these parameters, which are of practical significance and whose agreement with experiments provide a first estimation of how well the model performs, are shown in Table 1.

Thirdly, there exists a significant number of numerical simulations for this case that had virtually become a benchmark, because of its tractability on available computational facilities not only with Reynolds Averaged Navier-Stokes (RANS) or unsteady RANS, URANS (Young and Ooi, 2007), but even with Large Eddy Simulations, LES (Kravchenko and Moin, 2000; Young and Ooi, 2007) and Direct Numerical Simulations, DNS (Ma et al., 2000). These simulations clearly demonstrate that LES and DNS calculate effectively the flow case of $\operatorname{Re}_{\mathrm{D}}=3900$, provided that the basic characteristics of the simulations have been correctly selected. These characteristics, whose values for these and the present simulations are also shown in Table 1, are the normalized dimensions in the stream-wise (x), cross-flow (y) and vertical (z) directions $L_{x} / D, L_{y} / D$ and $L_{z} / D$, respectively, the total number of control volumes $\left(N_{\text {total }}\right)$, the resolution in the vertical direction $\left(n_{z}\right)$, the normalized time step $\left(U_{\infty} d t / D\right)$, where $d t$ is the time step used, and the normalized averaging time, i.e. the number of shedding periods $\left(N_{T}\right)$ that is employed for the derivation of time-average values. However, LES require very large computational storage, power and CPU time; the corresponding requirements for DNS are much higher.

With the objective to exploit the advantages of LES in reduced required computational time, the hybrid models have been developed. One of these models is the Shear Stress Transport Scale Adaptive Simulation model, SST-SAS (Menter and Egorov, 2008) that is based on the SST model (Menter, 1994), which has already been applied successfully in air flows (Stamou and Katsiris, 2006). In the present work, we refer to this model simply as SAS; this model that is briefly described in section 2.2 combines standard RANS with an improved URANS mode for the steady and unsteady flow regions, respectively, and saves order of magnitudes of computing power (Widenhorn et al., 2008) by simulating boundary layers using RANS. Virtually, the SAS is an improved URANS formulation, with the ability to adapt the length scale to resolved turbulent structures. In order for the SAS method to produce a resolved turbulent spectrum, the underlying turbulence model has to go unsteady. This is typically the case for flows with a global instability, such as the flow with vortex interactions past a cylinder at $\operatorname{Re}_{D}=3900$. In the present work, we compare model predictions with experiments, URANS simulations and LES to evaluate the capacity of the proposed SAS model. Calculations were performed with the numerical code CFX (http://www.ANSYS.com) which employes the SAS model.

\section{Governing equations and turbulence models}

\subsection{Equations of the model}

Calculations were performed using the finite volume method; a 3-D grid is constructed to create finite volumes, which are used to conserve relevant quantities such as mass, momentum, and energy. The continuity (1) and momentum (2) equations are integrated over each control volume, in a discrete sense.

$$
\begin{aligned}
& \frac{\partial \rho}{\partial t}+\frac{\partial\left(\rho U_{i}\right)}{\partial x_{i}}=0 \\
& \frac{\partial\left(\rho U_{i}\right)}{\partial t}+\frac{\partial}{\partial x_{i}}\left(\rho U_{i} U_{j}\right)=-\frac{\partial p}{\partial x_{i}}+\frac{\partial}{\partial x_{i}}\left[\left(\mu+\mu_{t}\right)\left(\frac{\partial U_{j}}{\partial x_{i}}+\frac{\partial U_{i}}{\partial x_{j}}\right)\right]+S_{M}
\end{aligned}
$$

where $U_{i}$ is the velocity in the $i$ - direction of a Cartesian coordinate system, $\rho$ is the density of the fluid, $p$ is the pressure, $\mu$ and $\mu_{t}$ are the dynamic and eddy viscosities, respectively, and $S_{M}$ is the source term of the momentum conservation equation. 


\subsection{Turbulence model}

The hybrid SST-SAS turbulence model consists of the transport equations (3) and (4) for the turbulent kinetic energy $(k)$ and the turbulence eddy frequency $(\omega)$, respectively. The main difference of the SAS model from the $k-\omega$ based SST model (Menter, 1994) is the presence of the additional source term $Q_{\text {SAS }}$ that is described by equation (5).

$$
\begin{aligned}
& \frac{\partial \rho k}{\partial t}+\frac{\partial}{\partial x_{i}}\left(\rho U_{i} k\right)=\frac{\partial}{\partial x_{i}}\left[\left(\mu+\frac{\mu_{t}}{\sigma_{k}}\right) \frac{\partial k}{\partial x_{i}}\right]+P_{k}-\rho c_{\mu} k \omega \\
& \frac{\partial \rho \omega}{\partial t}+\frac{\partial}{\partial x_{i}}\left(\rho U_{i} \omega\right)=\frac{\partial}{\partial x_{i}}\left[\left(\mu+\frac{\mu_{t}}{\sigma_{\omega}}\right) \frac{\partial \omega}{\partial x_{i}}\right]+\alpha \frac{\omega}{k} P_{k}-\rho \beta \omega^{2}+\left(1-F_{1}\right) \frac{2 \rho}{\sigma_{\omega 2}} \frac{1}{\omega} \frac{\partial k}{\partial x_{i}} \frac{\partial \omega}{\partial x_{i}}+Q_{S A S} \\
& Q_{S A S}=\max \left[\rho \zeta_{2} K S^{2}\left(\frac{L}{L_{v K}}\right)^{2}-C \frac{2 \rho k}{\sigma_{\phi}} \max \left(\frac{1}{\omega^{2}} \frac{\partial \omega}{\partial x_{i}} \frac{\partial \omega}{\partial x_{i}}, \frac{1}{k^{2}} \frac{\partial k}{\partial x_{i}} \frac{\partial k}{\partial x_{i}}\right), 0\right]
\end{aligned}
$$

$F_{1}$ is a blending function that switches smoothly between the RANS and the improved URANS (LES-like) mode based on local solution values and the distance from the wall, $\mathrm{P}_{\mathrm{k}}$ is the turbulence production term that is equal to $P_{k}=\mu_{t} S^{2}$, where $S$ represents the first velocity derivative $\left(U^{\prime}\right), L$ is the turbulence length scale, and $L_{v k}=K S /\left|U^{\prime \prime}\right|$ is the von Karman length scale, where $\mathrm{k}$ is the von Karman constant and $U^{\prime \prime}$ is the second velocity derivative. The values of the model parameters are $c_{\mu}=0.09, \sigma_{k}=1.18, \sigma_{\omega}=2.0$, $\sigma_{\omega 2}=1.17, \alpha=5 / 9, \beta=0.075, \zeta_{2}=3.51, \sigma_{\phi}=2 / 3$ and $C=2.0$ (Menter and Egorov, 2008).

The selection of the mode (RANS or LES-like) is based on the argument made by Davidson, (2006), that in unsteady flow regions $L_{v k}$ values are smaller than in steady flow regions. Therefore, in unsteady flow regions, such as vortex shedding in wakes, where the improved LES-like mode is applied, $L_{v k}$ values are small, $\left(L / L_{v k}\right)^{2}$ values are large, $Q_{S A S}$ values are large (see equation 5$), \omega$ values are large (see equation 4$)$ and $\mu_{\mathrm{t}}$ values are low enough (because $\mu_{\mathrm{t}}$ is proportional to $\mathrm{k} / \omega$ ) to allow the development of small turbulent scales without any damping of the finest resolved turbulent fluctuations. In steady flow regions, i.e. in attached or mildly separated boundary layers, $\left(L / L_{v k}\right)^{2}$ values are small, $Q_{s A s}$ is equal to zero and the RANS model ( $k-\omega$ based SST) is applied.

\section{Numerical details}

\subsection{Computational domain}

The diameter and the height of the cylinder were equal to $D=0.10 \mathrm{~m}$ and $\mathrm{h}=0.10 \mathrm{~m}$, respectively. Moreover, the dimensions of the computational domain that is shown in Figure 1 were equal to $L_{x}=30 D$, $L_{y}=11 D$ and $L_{2}=h=1 D$. We have selected these dimensions to be close to the lower limits of their ranges of values found in the literature (see Table $1 ; L_{x}=20 \div 65 \mathrm{D}, L_{y}=10 \div 50 \mathrm{D}$ and $L_{z}=0.5 \pi \div 2 \pi \mathrm{D}$ ) to reduce the computational time; the required vertical dimension and resolution (see section 3.3) have been chosen based on the prior knowledge of the sizes of the stream wise vortices past the cylinder (Kravchenko and Moin, 2000; Ma et al., 2000), whose wavelength were estimated equal to 0.4D close to the cylinder and equal to $D$ further downstream.

\subsection{Boundary conditions}

Boundary conditions were defined at the boundaries of the computational domain, i.e. the inlet, the outlet, the sides, the bottom and the top. In the present work, we have implemented the most frequently encountered boundary conditions that are shown in Figure1. At the inlet a uniform normal velocity was defined equal to the free stream velocity $\left(U_{\infty}\right)$; this value was set equal to $U_{\infty}=0.0429 \mathrm{~m} \mathrm{~s}^{-1}$ to produce a Reynolds number equal to $R_{D}=U_{\infty} D / v=3900$, where $v=1.1 \times 10^{-6} \mathrm{~m}^{2} \mathrm{~s}^{-1}$ is the viscosity of the water. At the outlet boundary the average static pressure was set equal to $0 \mathrm{~Pa}$. The sides were considered as free slip walls, while at the top and bottom boundaries a periodic condition was imposed, which is the same that was applied by the majority of researchers (Kravchenko and Moin, 2000; Young 
and Ooi, 2007); periodicity in the z-direction implies that the cylinder has infinite height and is frequently used in order to reduce the size of the computational domain.

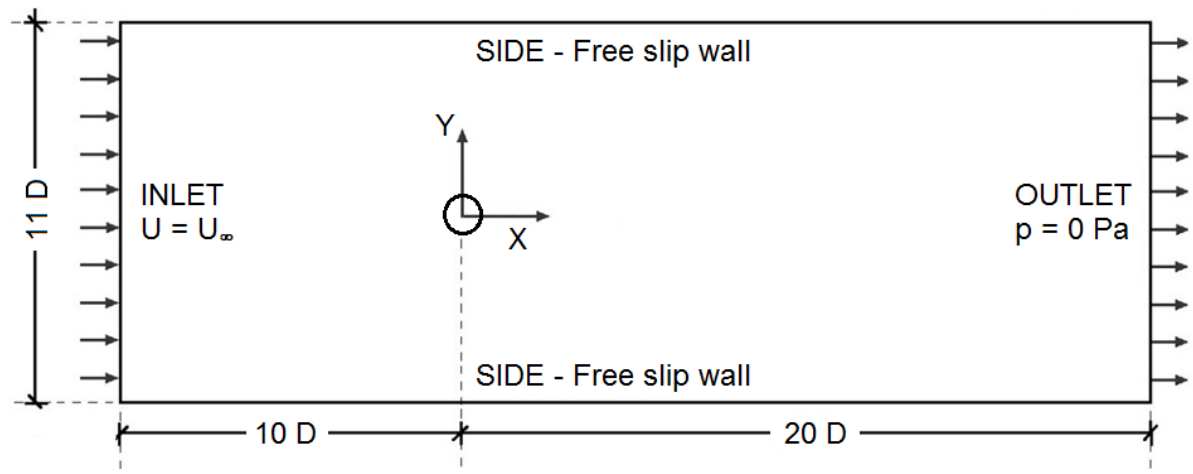

Figure 1. Computational domain and boundary conditions

\subsection{Numerical grids, grid independence tests and convergence criteria}

To ensure grid independent results, calculations were performed for three unstructured numerical grids of different density that were named "coarse", "medium" and "fine" and consisted of $\mathrm{N}_{\text {total }}=0.38,1.47$ and $2.51 \times 10^{6}$ elements, respectively. The minimum and maximum element edge size was equal to $2 \mathrm{~mm}$ (0.2 D) and $20 \mathrm{~mm}$ for the coarse grid, and $1 \mathrm{~mm}$ and $10 \mathrm{~mm}$ for the medium and fine grid. We have selected the number of vertical elements equal to $n_{2}=5$ for the coarse grid and 10 for the medium and fine grid; these values are close to the lower limits of the range of values applied by various researchers $\left(n_{2}=4 \div 128\right)$. The main difference of the medium from the fine grid is that the latter had a more detailed resolution (i.e. $70 \%$ more elements), mainly in the region near the surface of the cylinder. A detail of the medium grid in the vicinity of the cylinder is presented in Figure 2; the grid has been refined close to the surface of the cylinder in order to resolve the wall shear layers and capture accurately the large velocity gradients in the boundary layer region, thus avoiding drag production due to grid coarseness.

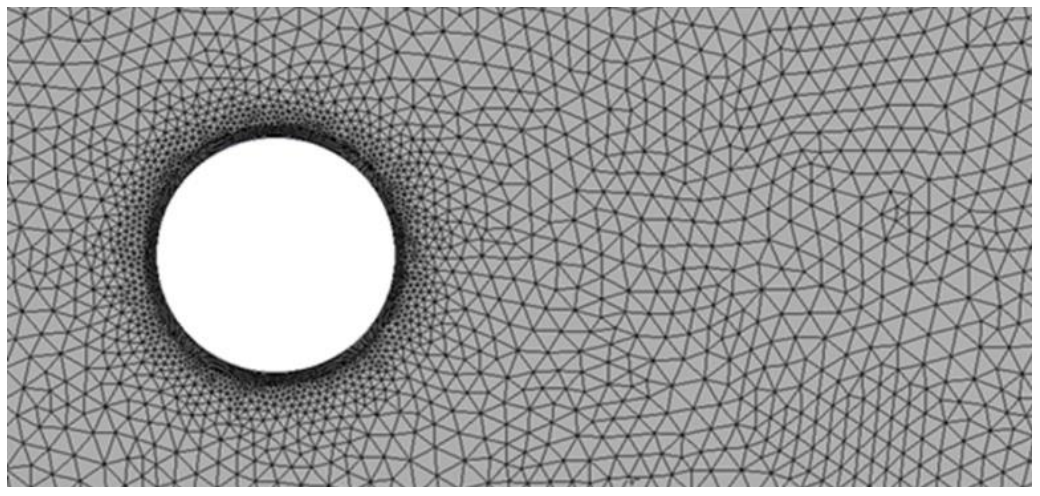

Figure 2. Horizontal section of the medium numerical grid; detail near the cylinder

In Figure 3 some indicative profiles of average and turbulent flow characteristics are shown, which depict that grid independent results were ensured for the medium grid; thus, the presentation and discussion of the results that follows refers to this grid. A similar behaviour was observed in grid independence URANS calculations. Regarding the convergence criteria, the Root Mean Square (RMS) residuals of $p, U_{i}, k$ and $\omega$ were monitored and calculations were not stopped before their values were lower than $10^{-5}$. 

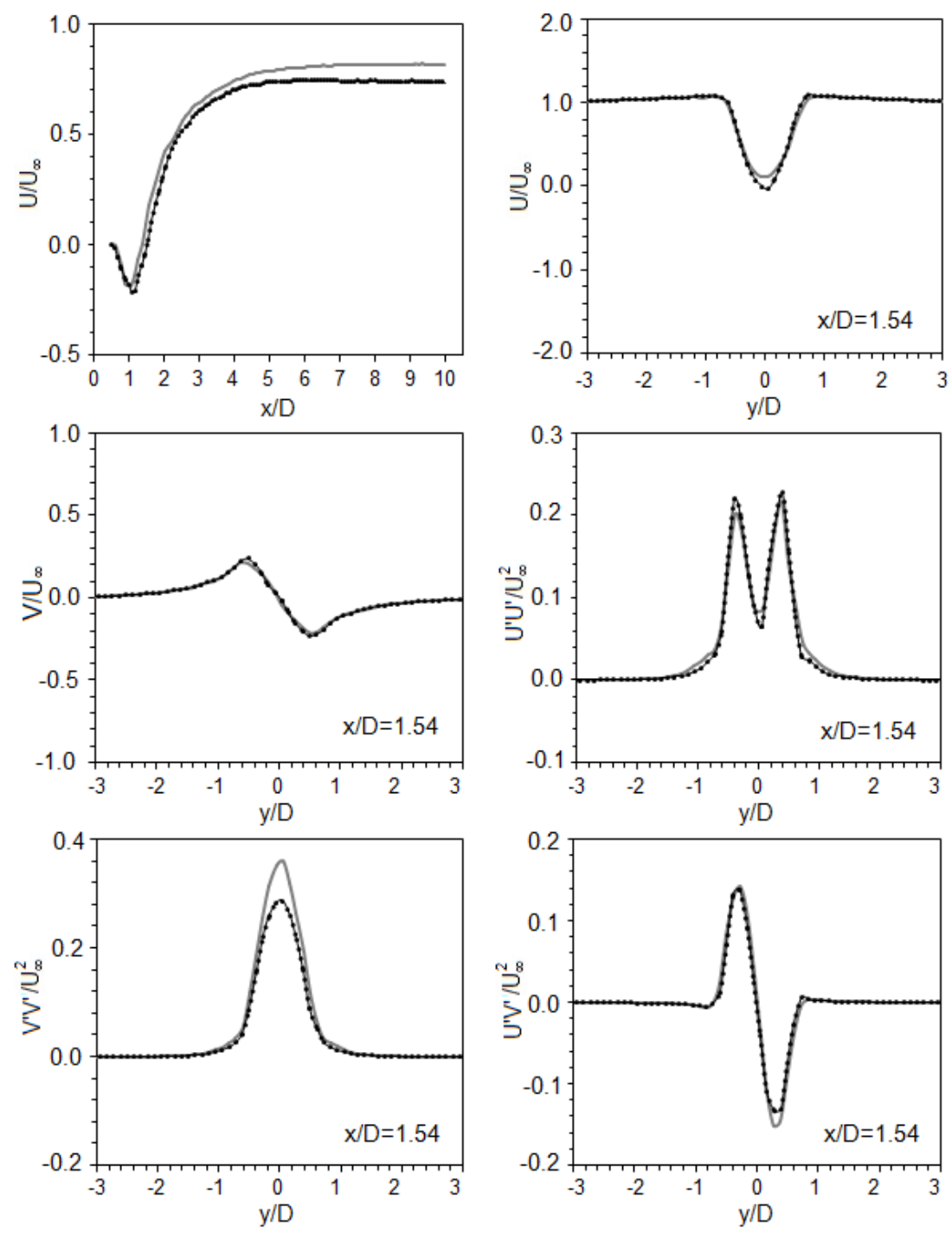

Figure 3. Effect of the size of the grid on the distribution of various mean and turbulent quantities; Coarse; Medium; Fine

\subsection{Initial and start-up conditions}

Firstly, we performed RANS calculations using the $k-\omega$ based SST model (Menter, 1994) to determine the steady state flow field that was used as initial condition for the SAS calculations; RANS calculations were executed for an initial period until the start-up transients had been transported through the computational domain and the flow had evolved to steady shedding. This can be clearly seen in Figure 4, in which the time history of the drag coefficient $\left(C_{D}\right)$ is shown. After this initial period, the SAS calculations were continued for another $\mathrm{N}_{\mathrm{T}}=30$ shedding periods $(\mathrm{T})$ on which the results were averaged; this averaging time is identical to the values used by the majority of the researchers $\left(\mathrm{N}_{\mathrm{T}}=10-86\right)$. Moreover, two additional runs were made (see Table 1) for the medium grid for $\mathrm{N}_{\mathrm{T}}=20$ and 10 to examine the effect of $\mathrm{N}_{\mathrm{T}}$. 


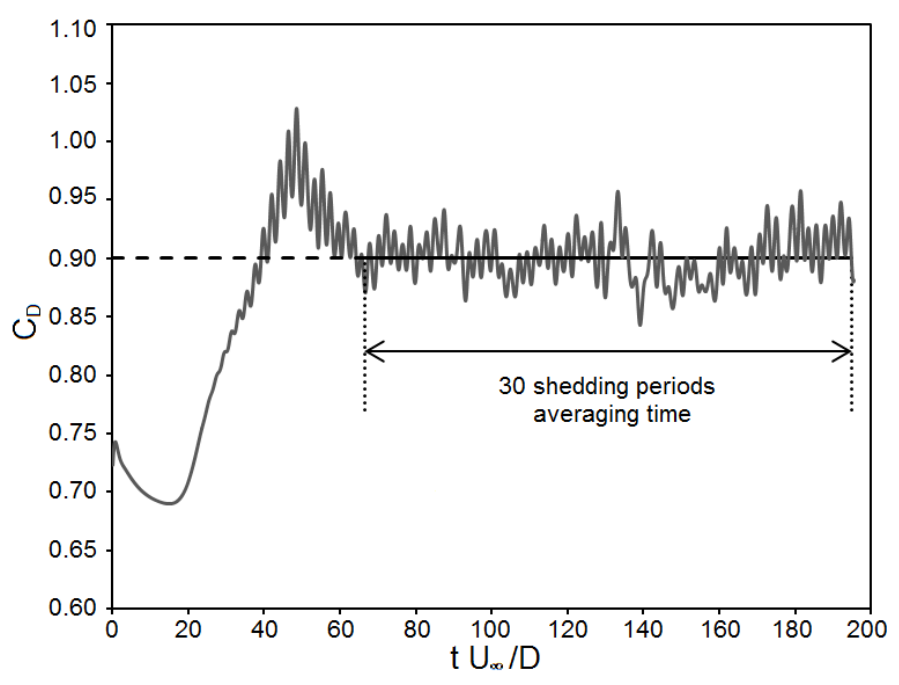

Figure 4. Time history of the drag coefficient

\section{Results and discussion}

To evaluate the capacity of the proposed SAS model, we compare model predictions with the experimental data that have been used for model assessment by the majority of the researchers; these are the PIV experiments by Lourenco and Shih (1993) near the wake of the cylinder $(x / D<3.0)$ and the HWA velocity measurements by Ong and Wallace (1996) outside the recirculation zone $(3<x / D<10)$. Moreover, the calculated distribution of the pressure coefficient along the surface of the cylinder is compared with the measurements of Norberg (1994).Calculated flow quantities mainly with SAS, but also with URANS, were averaged in the z-direction, as in the cases of very good LES by Kravchenko and Moin (2000) and Young and Ooi (2007), with which the present simulations were compared.

\subsection{The effect of SAS}

In Figure 5 an instantaneous plot of the vorticity magnitude $\left(\omega D / U_{\infty}\right)$ is shown for URANS and SAS calculations. The predicted flow by URANS with $\mathrm{n}_{2}=10$ exhibits a 2-D, regular vortex shedding, while SAS calculations with $\mathrm{n}_{\mathrm{z}}=10$ produce a 3-D irregular vortex shedding that is similar to LES vorticity plots (Kravchenko and Moin, 2000; Young and Ooi, 2007); this behaviour is due to the more dissipative character of URANS compared to SAS; a more detailed discussion is performed in section 4 (see also Figure 16). It should also be pointed out that SAS with $\mathrm{n}_{2}=5$ exhibits some three-dimensionality but with minor development of the smaller turbulent scales in the far wake of the cylinder; this is attributed to the coarseness of the grid in the z-direction.

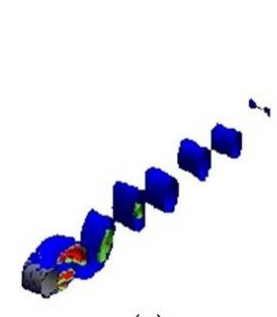

(a)

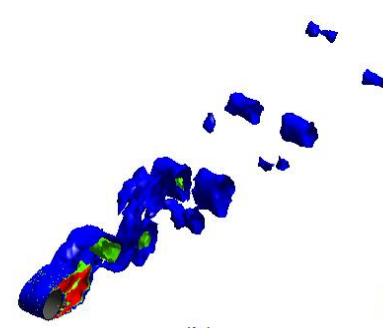

(b)

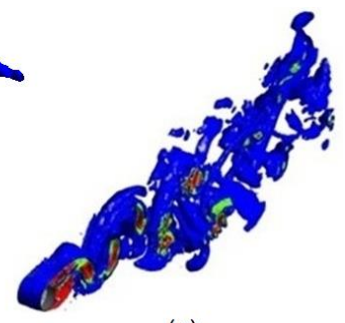

(c)

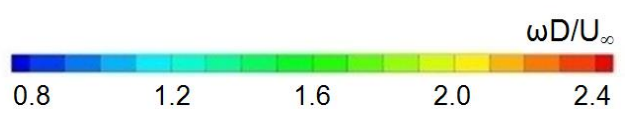

Figure 5. Instantaneous plots of vorticity magnitude using (a) 3D URANS, $n_{z}=10$,(b) SAS, $n_{z}=5$ and (c) SAS, $n_{z}=10$ 
Figure 6 depicts an instantaneous view of the steady (RANS) and unsteady (LES-like) regions in a part of the computational domain, which are characterized by the blending function $\left(F_{1}\right)$ that denotes the blending of two turbulence models; values of 0 and 1 correspond to LES-like and RANS, respectively. Figure 6 shows that in the wake of the cylinder the turbulence model turns unsteady in order to capture the intensive velocity fluctuations; in this region vortices initiating from the cylinder are shed and the small alternating turbulence scales activate the LES-like mode to produce the vorticity distribution shown in Figure $5 \mathrm{~b}$. Thus, URANS simulation produces only the large-scale unsteadiness, whereas the LES-like mode adjusts to the already resolved scales in a dynamic way and allows the development of a turbulent spectrum in the detached regions.

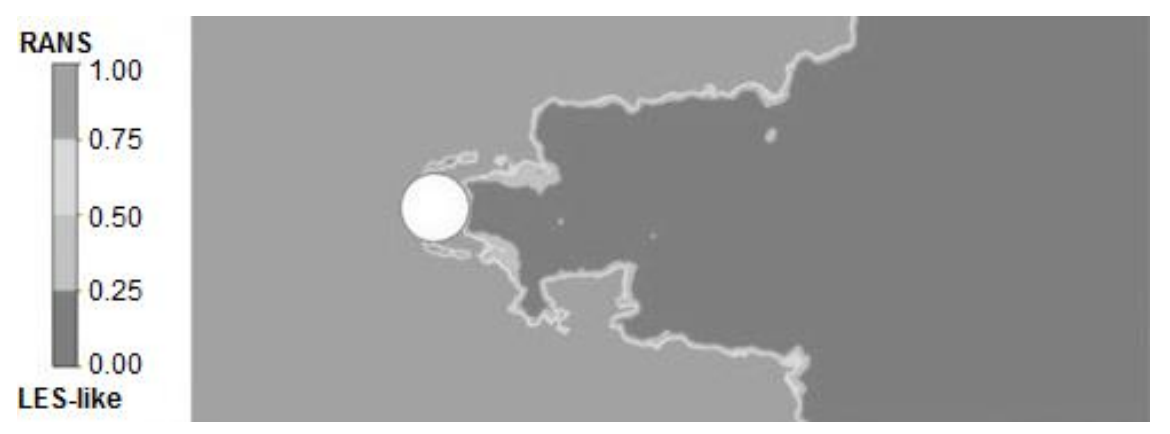

Figure 6. Instantaneous view of the steady and unsteady regions in the computational domain at a horizontal plane at $\mathrm{z} / \mathrm{D}=0.5$; values of the blending function for LES-like and RANS behaviour

\subsection{Overall flow parameters}

The values of the overall flow parameters (see section 1) are shown in Table 1 together with experimental values and LES and URANS predictions from other researchers.

1. Distribution of pressure (p). The calculated distributions of normalized pressure, i.e. pressure coefficient $\left(C_{p}\right)$, in a horizontal plane and along the surface of the cylinder, are shown in Figure 7 and Figure 8, respectively; in Figure 8 experimental values (Norberg, 1994) and computations with LES (Kravchenko and Moin, 2000; Young and Ooi, 2007) are also shown. The pressure coefficient $\left(C_{p}\right)$ is defined as

$$
C_{p}=\frac{p-p_{\infty}}{\frac{1}{2} \rho U_{\infty}^{2}}
$$

where $p$ is the actual pressure and $p_{\infty}$ is the pressure in the free stream region. Figure 8 shows that pressure takes its maximum value at the upstream stagnation point; then, it is reduced down to a minimum value, after which it starts to increase until it reaches a constant value $\left(p=p_{b}\right)$ that is the "base pressure", which is expressed in non-dimensional formulation $\left(\mathrm{C}_{\mathrm{Pb}}\right)$ as follows

$$
C_{p b}=\frac{p_{b}-p_{\infty}}{\frac{1}{2} \rho U_{\infty}^{2}}
$$

Figure 8 depicts that SAS predict satisfactorily the pressure distribution on the surface of the cylinder; thus, the forces on the cylinder are expected to be well predicted. Moreover, the calculated base pressure $\left(-\bar{C}_{p b}=0.93\right)$ that is a good indicator of whether the pressure in the wake is correct lies within the experimental range of values (0.83-0.93). Taking into account that at $R_{D}=3900$ the largest contribution to drag is the pressure drag arising from the wake downstream of the cylinder (Fairbanks, 1998), the overall drag is expected to be well predicted by the SAS. As expected, the distribution of pressure with URANS coincides with that of SAS up to $\Theta=120^{\circ}$, because according to Figure 7 URANS is used in this region by both models. 


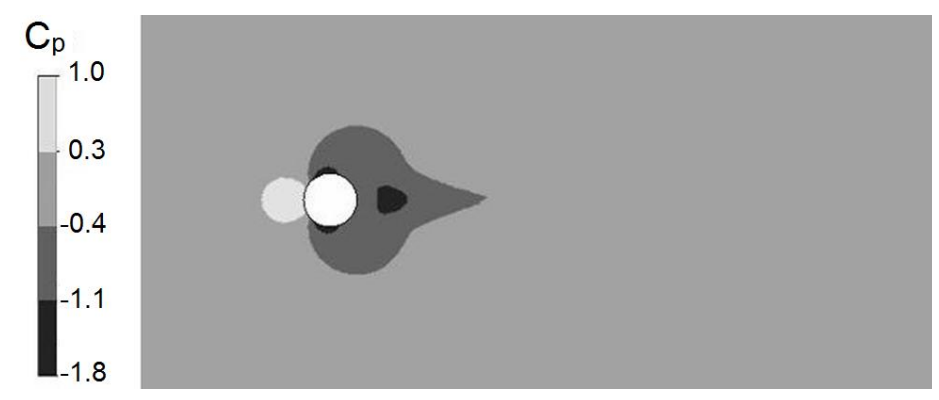

Figure 7. Distribution of pressure coefficient near the cylinder at a horizontal plane at $z / D=0.5$

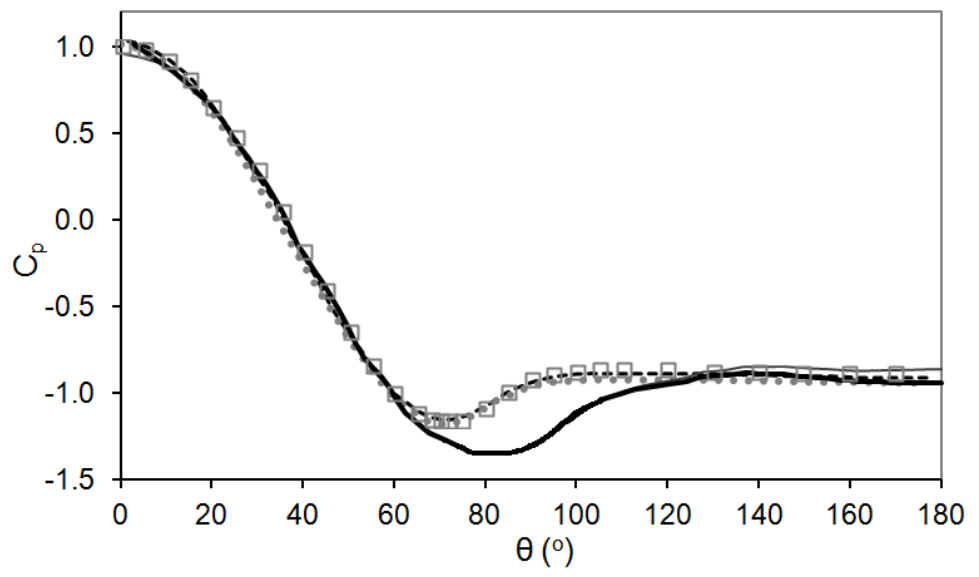

Figure 8. Distribution of pressure coefficient along the surface of the cylinder;

$\square$ experiments (Norberg, 1994); …... LES (Kravchenko and Moin, 2000);

- - - LES (Young and Ooi, 2007); — 3D URANS; — SAS

2. Total drag force. The total drag force acting on the cylinder surface in the axial direction is the sum of the drag force caused by pressure differences on either side of the cylinder, and the friction drag caused by shear stresses (Fairbanks, 1998). The average value of the drag coefficient $\left(C_{D}\right)$ was determined by equation (8)

$C_{D}=\frac{F_{D}}{\frac{1}{2} \mathrm{DhU}_{\infty}^{2}}$

equal to $C_{D}=0.90$ and is in satisfactory agreement with the experimental values (0.93-1.03); the time history of $C_{D}$ is shown in Figure 4.

3. Recirculation region. The adverse pressure gradient in the rear half of the cylinder deters the flow from surrounding its surface since it decreases the initial pressure gradient that forces the flow to the back. Consequently, the boundary layers separate from the wall forming a highly turbulent region of low pressure and a recirculation region of length equal to $L_{\text {rec }}$ that was calculated equal to $L_{\text {rec }} / D=1.10$; this value is in satisfactory agreement with the range of experimental values $(1.13 \div 1.53)$.

4. Period of shedding. In the wake region of the cylinder the flow separation tends to roll up the flow into swirling eddies, resulting in alternate shedding of vortices with frequency $f$ (or period $T=1 / f$ ) that is expressed in non-dimensional form as Strouhal number (St)

$\mathrm{St}=\frac{\mathrm{D}}{\mathrm{U}_{\infty} \mathrm{T}}=\frac{\mathrm{f} \cdot \mathrm{D}}{\mathrm{U}_{\infty}}$

The period was calculated equal to $\mathrm{T}=10.3 \mathrm{~s}$ and $\mathrm{St}=0.228$, a value that is very close to the experimental range of values $(0.21 \div 0.22)$. 
Table 1 depicts that the agreement of the SAS predicted values of the overall flow parameters with measurements is satisfactory; thus, the SAS is expected to perform well; however, not as good as LES. Moreover, (i) the decrease of the number of shedding periods for the averaging from $\mathrm{N}_{\mathrm{T}}=30$ to 20 and 10 does not have a significant effect on the values of the overall flow parameters, and (ii) the SAS results with the coarse grid are also satisfactory; in fact they show a better agreement with measurements, which can be characterized as fortuitous. Furthermore, URANS calculations show also a satisfactory agreement with measured values, as in the case of URANS results by Ayyappan and Vengadesan (2008), who used a longer vertical domain $\left(L_{z}=4 D\right)$ with significantly higher resolution $\left(n_{z}=20 \div 38\right)$ to predict longer recirculation lengths $\left(L_{\text {rec }} / D=1.20 \div 1.75\right)$.

\subsection{Mean flow characteristics}

The calculated distribution of the normalized mean stream-wise velocity $\left(U / U_{\infty}\right)$ along the centerline $(y=0)$ downstream of the cylinder, which is plotted in Figure 9, shows a satisfactory agreement with experimental data that is almost of the same quality as in LES calculations. Generally, predicted velocities close to the cylinder $(x / D<3.5)$ are higher than measurements, while far from the cylinder velocities are lower than measurements. For $x / D<1.6$ negative velocity values are observed with an absolute maximum equal to 0.23 that is within the range of experimental values $(0.23 \div 0.25)$. Downstream of the recirculation area, the flow accelerates until the velocity reaches a constant value that is approximately equal to the 0.75 of the free stream velocity $\left(U_{\infty}\right)$ showing again a very satisfactory agreement with the experimental value.

The calculated cross-stream distributions of the normalized mean stream-wise $\left(U / U_{\infty}\right)$ and cross-flow velocities $\left(V / U_{\infty}\right)$ that are plotted in Figure 10 and 11 , respectively, exhibit the expected trend, i.e. (i) the profiles of $\mathrm{U} / \mathrm{U}_{\infty}$ are symmetrical showing a $\mathrm{V}$-shape near the cylinder, like the experiments (Lourenco and Shih, 1993), (ii) the profiles of $\left(\mathrm{V} / \mathrm{U}_{\infty}\right)$ are anti-symmetrical, (iii) the wake mean stream-wise velocities increase following the spreading of the wake. The predicted stream-wise velocities are higher than measurements near the cylinder and lower than measurements far from the cylinder; this behaviour is consistent with the comparison of centerline velocity in Figure 9.

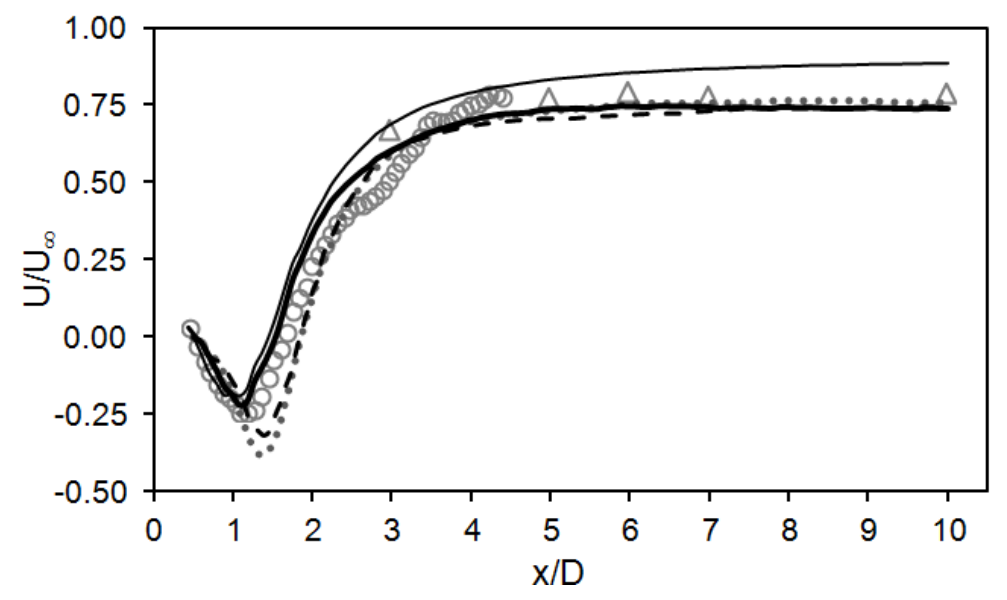

Figure 9. Calculated mean stream-wise velocity along the centerline of the wake;

$\circ$ experiments (Lourenco and Shih, 1993); $\triangle$ experiments (Ong and Wallace, 1996) $\cdots . . .$. LES

(Kravchenko and Moin, 2000); - - - LES (Young and Ooi, 2007); — 3D URANS; — SAS

The cross-flow velocities near the cylinder are under-predicted close to the centerline and are higher than the measured values far from the centerline, thus showing more mixing in the cross-stream direction. Generally, the predicted SAS mean velocity profiles show a satisfactory agreement with experiments, which as expected is not as good as the agreement with the LES calculations. Figure 10 and Figure 11 also depict that close to the cylinder $(x / D=1.06)$ URANS distributions practically coincide with the corresponding SAS profiles; then, they are more uniform in the cross-stream direction, a behaviour that can be attributed to the dissipative character of URANS (You and Kwon, 2010). 


\subsection{Turbulent characteristics}

The calculated cross-stream distributions of the mean normalized Reynolds stresses, stream-wise ( $\left.U^{\prime} U^{\prime} / U_{\infty}^{2}\right)$, cross-flow $\left(V^{\prime} V^{\prime} / U_{\infty}^{2}\right)$ and shear $\left(U^{\prime} V^{\prime} / U_{\infty}^{2}\right)$, that are plotted in Figures 12, 13 and 14, respectively, exhibit the expected trend, i.e. (i) the profiles of $U^{\prime} U^{\prime} / U_{\infty}^{2}$ and $V^{\prime} V^{\prime} / U_{\infty}^{2}$ are symmetrical (Lourenco and Shih, 1993), (ii) the profiles of $U^{\prime} V^{\prime} / U_{\infty}^{2}$ ' are anti-symmetrical, (iii) the peaks of the profiles decrease and (iv) the separation of the $U^{\prime} V^{\prime} / U_{\infty}^{2}$ peaks increase following the spreading of the wake.

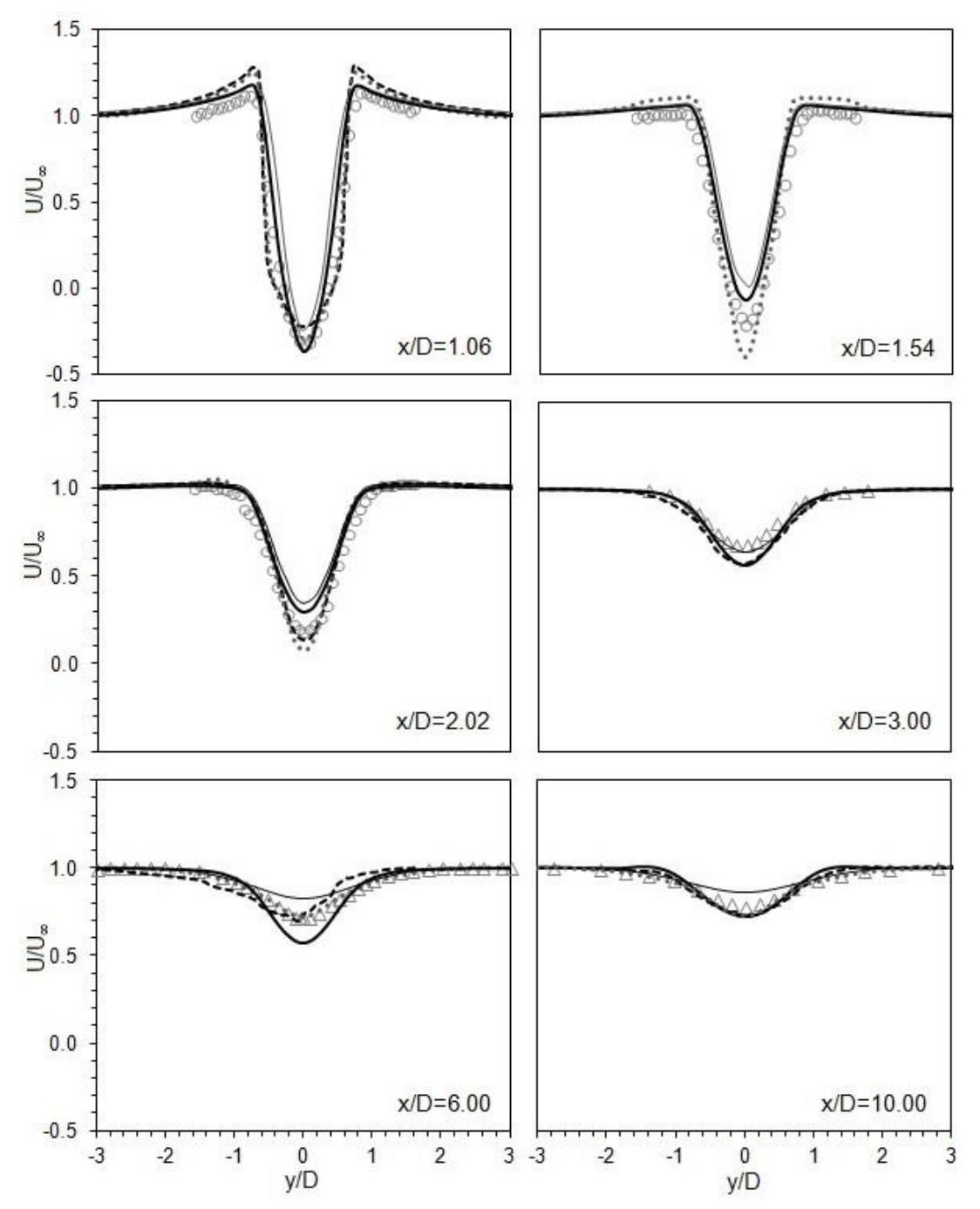

Figure 10. Calculated mean stream-wise velocities in the wake of the cylinder at various locations;

$\circ$ experiments (Lourenco and Shih, 1993); $\triangle$ experiments (Ong and Wallace, 1996); …... LES

(Kravchenko and Moin, 2000); - - - LES (Young and Ooi, 2007); — 3D URANS; — SAS

Generally, near the cylinder ( $x / D<4.0)$ SAS profiles show a satisfactory agreement with experiments; for example the (absolute) peaks of all three profiles and the separation of peaks in the $U^{\prime} V^{\prime} / U_{\infty}^{2}$ profiles agree quite well with experiments. This satisfactory agreement is observed also in $V^{\prime} V^{\prime} / U_{\infty}^{2}$ for $x / D \leq 2.02$ (Figure 13) and in $U^{\prime} V^{\prime} / U_{\infty}^{2}$ for $x / D \leq 4.00$ (Figure14), where LES are not reported. However, far from the cylinder the agreement of $U^{\prime} U^{\prime} / U_{\infty}^{2}$ and $U^{\prime} V^{\prime} / U_{\infty}^{2}$ is not very satisfactory; the peaks are lower and their separation is wider than the experimental values. Indicatively, calculated $U^{\prime} U^{\prime} / U_{\infty}^{2}$ distributions for $x / D>6.0$, that are plotted in Figure12, show exactly the same behaviour with the LES of Kravchenko and Moin (2000) using the low order upwind scheme; this behaviour is characterized by the 
under prediction of the peak values of $U^{\prime} U^{\prime} / U_{\infty}^{2}$, which may be due to the presence of some additional diffusion in the SAS computations.
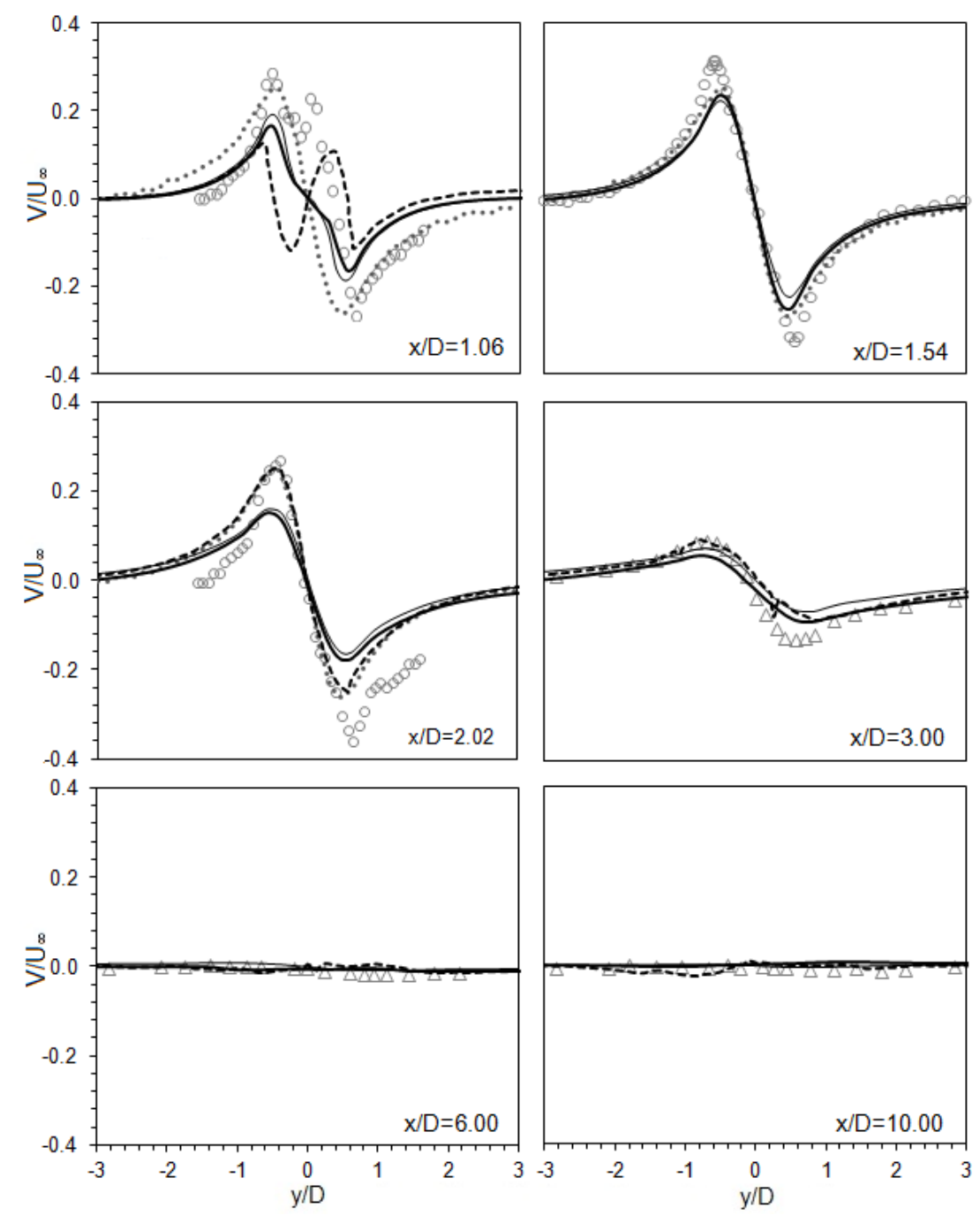

Figure 11. Calculated mean cross-flow velocities in the wake of the cylinder at various locations; $\circ$ experiments (Lourenco and Shih, 1993); $\triangle$ experiments (Ong and Wallace, 1996); $\cdots \cdot \cdots$ LES (Kravchenko and Moin, 2000); - - - LES (Young and Ooi, 2007); — 3D URANS; — SAS

We have attempted to identify the cause of this discrepancy based on the existing numerical studies and the relevant theory; if this cause is numerical diffusion, it cannot be due to the order of the differencing scheme (Kravchenko and Moin, 2000) that is relatively high in the SAS calculations, to the coarseness of the utilized numerical grid (Kravchenko and Moin, 2000) that is relatively fine, or to the resolution in the vertical direction that is adequate (Kravchenko and Moin, 2000; Ma et al., 2000). However, it can be due to the size of the vertical domain $\left(L_{2}=D\right)$ that we selected to be relatively small (see section 3.1 ) in order to reduce the computational cost. Kravchenko and Moin (2000) used $L_{2} / D$ values equal to $0.5 \pi$ and $\pi$; moreover, they commended that the small size of the vertical domain does not alter significantly the statistics in the near field wake $(x / D<2)$, but it is not adequate to capture important stream-wise vortices far from the cylinder. Ma et al. (2000) used $L_{z} / D=0.5 \pi, \pi$ and $2 \pi$ and observed that with increasing height of the vertical domain (while keeping the relevant vertical resolution $n_{z} / L_{2}$ constant) the streamwise velocity profiles become wider (i.e. more U-shaped), the recirculation length increases, and the peak values of the stream-wise stresses increase. 

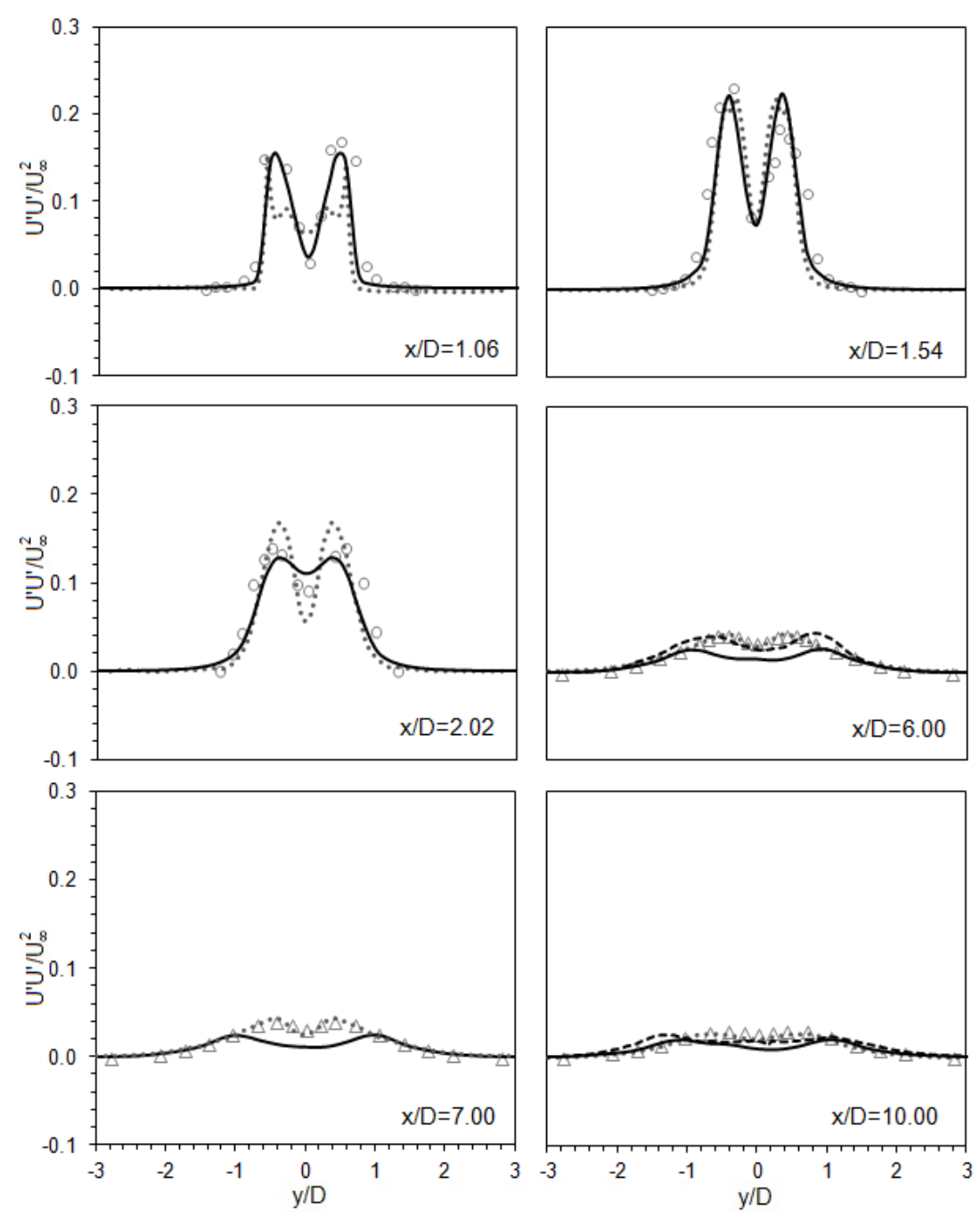

Figure 12. Calculated mean stream-wise normal Reynolds stresses in the wake of the cylinder at various locations; $\circ$ experiments (Lourenco and Shih, 1993); $\triangle$ experiments (Ong and Wallace, 1996);

...... LES (Kravchenko and Moin, 2000); - - - LES (Young and Ooi,2007); — SAS

To investigate the effect of the size of the vertical domain, we examined firstly the formation of vortices downstream of the cylinder by calculating the vorticity magnitude $\left(\omega \mathrm{D} / \mathrm{U}_{\infty}\right)$ and plotting its contours and iso-surfaces in Figure 15 and Figure 16, respectively (see also Figure 5). In these figures we have also included the corresponding LES calculations by Young and Ooi (2007) with a vertical length of the computational domain equal to $L_{2}=\pi D$ and various values of vertical resolution ranging from $n_{z}=4$ to 48 . Figures 15 and 16 show the appearance of smaller scales and the lengthening of the shear layer as vertical resolution increases; in the LES the flow field for $n_{z}=4$ is essentially 2-D, while for $n_{z}=16$ it shows some three-dimensionality, although on a larger scale than the eddies exhibited by the $n_{z}=32$ and $n_{z}=48$ cases. Similar results can be seen in Kravchenko and Moin (2000) and Ma et al. (2000). The SAS calculations that were performed using $n_{z}=10$ show a similar appearance with the $n_{z}=4$ case of Young and Ooi (2007); i.e. some three-dimensionality is evident, that is further reduced by the relatively small applied vertical length of the computational domain. Therefore, the present SAS calculations with $\mathrm{L}_{2}=\mathrm{D}$ and $n_{2}=10$ do not exhibit a fully 3-D behaviour with the appearance of sufficient smaller scale structures that would preserve a level of dissipation; however, they do show some three dimensionality and some smaller scale structures. 

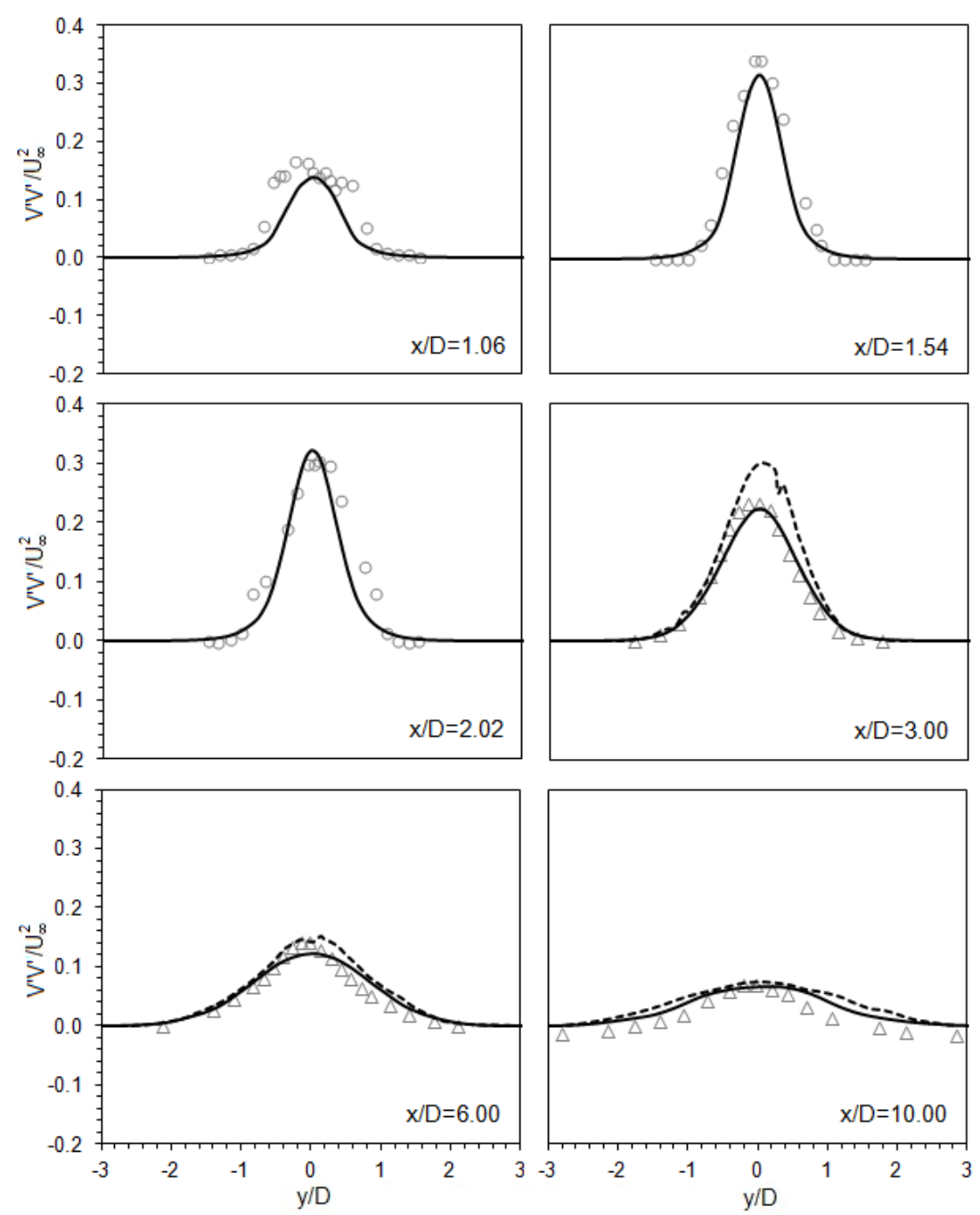

Figure 13. Calculated mean cross-flow normal Reynolds stresses in the wake of the cylinder at various locations; $\circ$ experiments (Lourenco and Shih, 1993); $\triangle$ experiments (Ong and Wallace, 1996);

$$
\text { - - - LES (Young and Ooi, 2007); — SAS }
$$

We have increased the size of the vertical dimension of the computational domain from $D$ to $\pi D$ using approximately the same resolution and repeated the calculations to check whether we would be able to improve the formation of vortices downstream of the cylinder; the size of the grid increased to $\mathrm{N}_{\text {total }}=3.69 \times 10^{6}$ elements. The effect on the vorticity plots of Figure 15 and Figure16 depict that the degree of three dimensionality increases and the SAS plot with $L_{2}=\pi D$ looks similar with the $n_{2}=16$ case of Young and Ooi (2007). However, the effect on the distributions of turbulent stresses far from the cylinder $(x / D \geq 6)$ was not important; profiles of stream-wise stresses remained practically unchanged, while the rest distributions showed only some minor improvement. It is worth noting that LES peak values of $U^{\prime} U^{\prime} / U_{\infty}^{2}$ by Young and Ooi (2007) showed a similar behaviour, i.e. they are smaller than experimental values, even for the case of $n_{2}=48$, which has very similar characteristics with the LES of Kravchenko and Moin (2000).

In order to investigate whether such under-prediction occurs systematically and to identify whether it is due to inherent numerical diffusion of the SAS or to other reasons, a more detailed analysis and comparison of SAS computations with LES is required, including applications also for other similar flow cases. Generally, the present calculations showed that SAS is an efficient and relatively fast turbulence modeling approach, especially in relevant practical problems, such as the modeling of the flow along a series of emerged or submerged cylinders that represent vegetation elements (Zhang et al., 2010), in 
which the very high accuracy that can be achieved by LES at the expense of large computational times is not required.
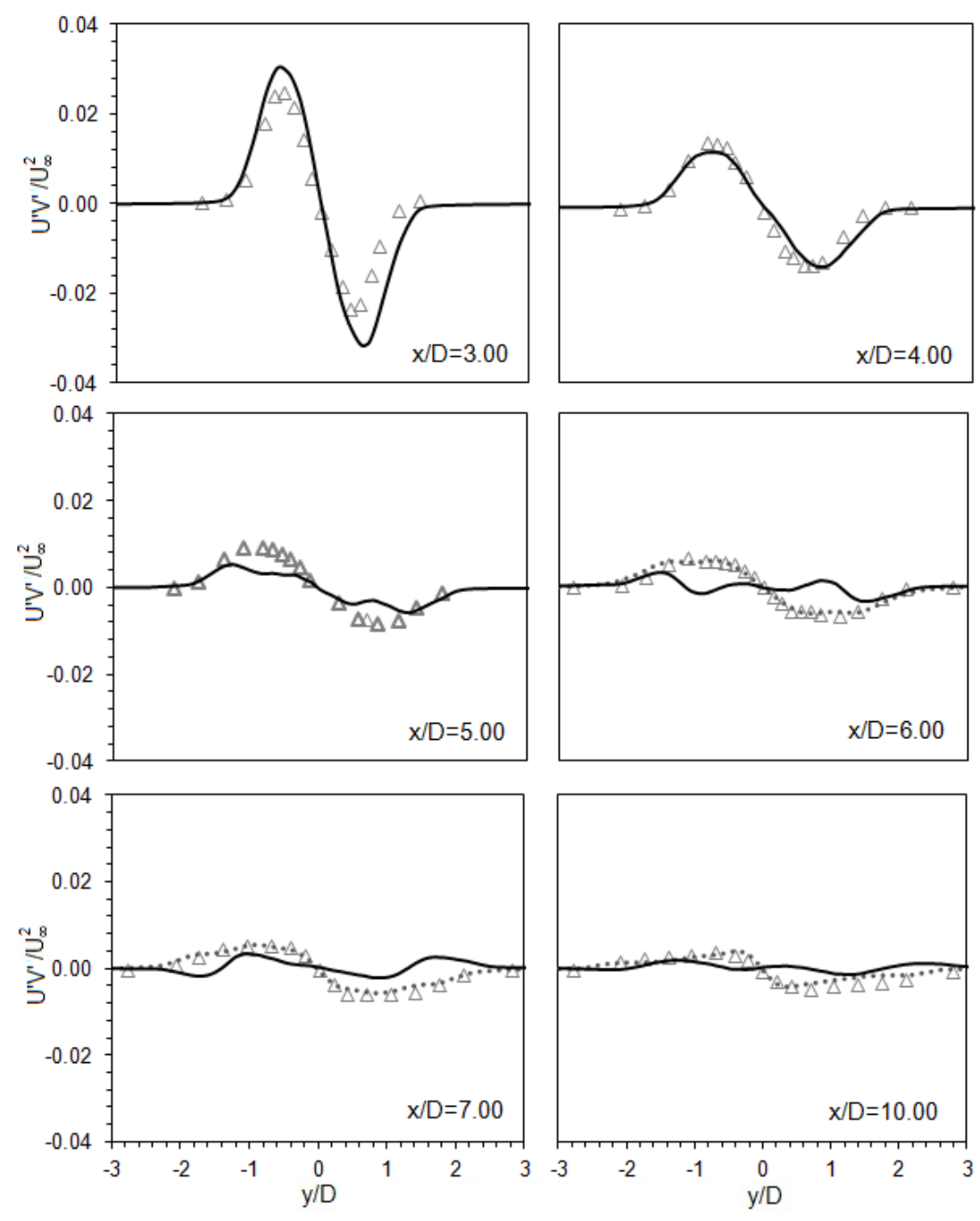

Figure 14. Calculated mean Reynolds shear stresses in the wake of the cylinder at various locations;

$\Delta$ experiments (Ong and Wallace, 1996); …... LES (Kravchenko and Moin, 2000); — - SAS

\subsection{Computational times}

Initially, we used in the calculations a time step equal to $\mathrm{dt}=0.005 \mathrm{~s}$ that corresponds to a nondimensional value equal to $U_{\infty} \mathrm{dt} / \mathrm{D}=0.002$, which is within the range of values used by other researchers $\left(0.001 \div 0.050\right.$ ); see also Table 1 . The maximum Courant number (based on $U_{\infty}$ and minimum element size of the medium grid) was equal to 0.21 . Then, to reduce the total CPU time we repeated the calculations using $d t=0.05 \mathrm{~s}$ and $\mathrm{U}_{\infty} \mathrm{dt} / \mathrm{D}=0.02$; the effect of this change on the results was not significant. 


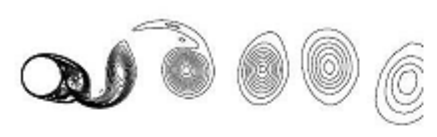

2D URANS

$\mathrm{L}_{2}=0, \mathrm{n}_{\mathrm{z}}=0$

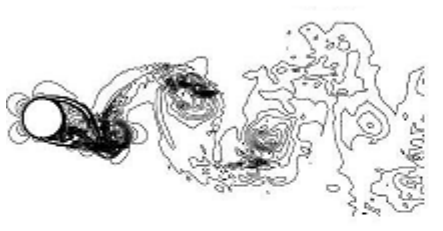

LES
$\mathrm{L}_{\mathrm{z}}=\pi \mathrm{m}, \mathrm{n}_{\mathrm{z}}=16$

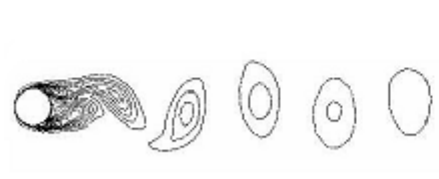

3D URANS

$L_{z}=D, n_{z}=10$

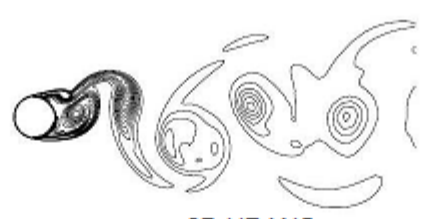

3D URANS

$L_{2}=\pi D, n_{z}=16$

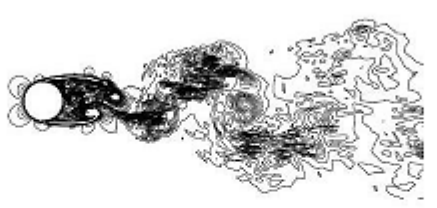

LES

$L_{2}=\pi D, n_{2}=32$

(a)

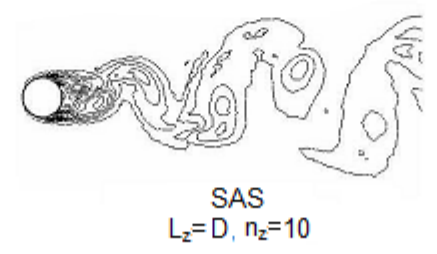

(b)
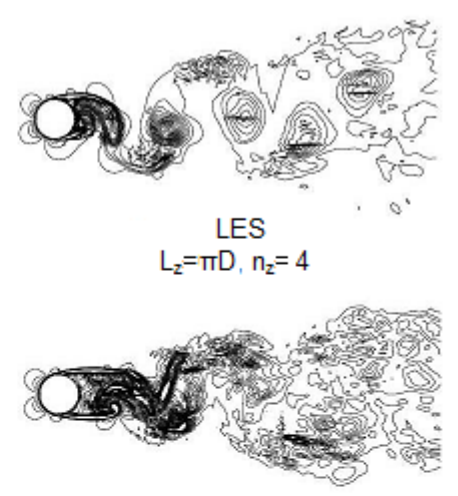

LES

$\mathrm{L}_{2}=\pi \mathrm{D}, \mathrm{n}_{2}=48$

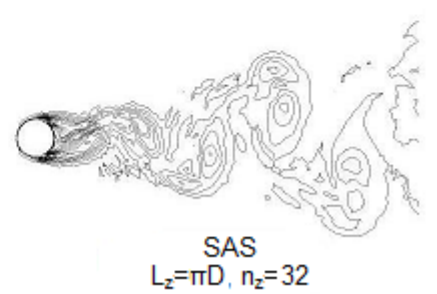

Figure 15. Contours of vorticity magnitude for $n_{z}=4,16,32$ and 48 by (a) Young and Ooi (2007) and (b) present 3D URANS and SAS calculations (16 contour levels between 0.5 and 10)

Table 2. Characteristics of computational speeds

\begin{tabular}{cccccc}
\hline Grid & $\mathrm{N}_{\text {total }}\left(\times 10^{6}\right)$ & $\mathrm{L}_{2} / \mathrm{D}$ & iterations $/ \mathrm{h}$ & Total runtime $(\mathrm{h})$ & CPU time $(\mathrm{h})$ \\
\hline Coarse & 0.380 & 1.0 & 188 & 32 & 193 \\
\hline Medium & 1.473 & 1.0 & 62 & 98 & 586 \\
\hline Fine & 2.509 & 1.0 & 53 & 115 & 690 \\
\hline Medium & 3.691 & $\pi$ & 38 & 159 & 957 \\
\hline
\end{tabular}

We have performed all calculations using a PC with 6 processors of $3.33 \mathrm{GHz}$. From the characteristics of the computation speeds for $\mathrm{dt}=0.05 \mathrm{~s}$ that are shown in Table 2, we note that the CPU time (CPU) for $\mathrm{N}_{T}=30$ shedding periods is equal to 586 hours and the required total computer (run) time is equal to 98 hours. The total CPU time (h) can be approximately correlated linearly with the size of the grid $\mathrm{N}_{\text {total }}$ $\left(\times 10^{6}\right)$ via equation (10); this linear relationship can also be observed in the LES computations of Young and Ooi (2007), in which the constant was equal to 427.7 .

$\mathrm{CPU}=278.9 \cdot \mathrm{N}_{\text {total }}\left(\times 10^{6}\right)$

We have made two additional runs for the medium grid using $\mathrm{N}_{\mathrm{T}}=20$ and 10 to examine the effect of $\mathrm{N}_{\mathrm{T}}$. Contrary to the findings of Franke and Frank (2002), calculations showed that the effect of $N_{T}$ is not significant and a minimum number of $\mathrm{N}_{\mathrm{T}}=10$ shedding periods is sufficient to produce values of overall flow parameters that are practically independent of $\mathrm{N}_{\mathrm{T}}$ (see Table 1); this remark is very important, because it results in the reduction of the required total computer time to less than 1.5 days. 


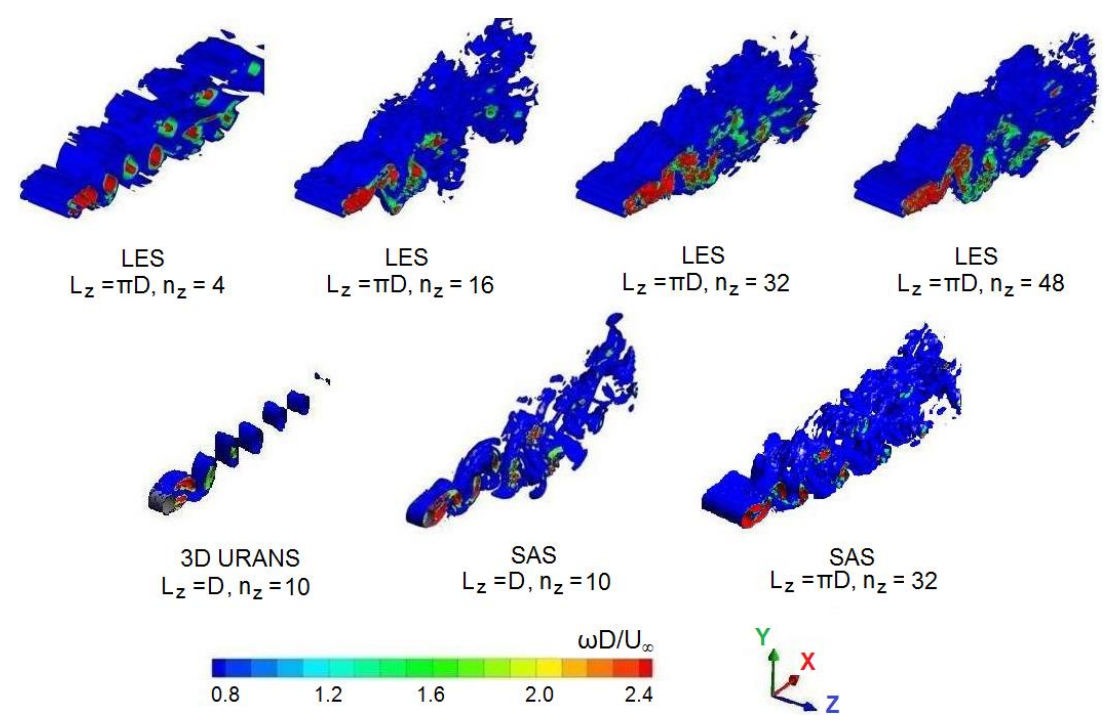

Figure 16. Isosurfaces of vorticity magnitude for $n_{2}=4,16,32$ and 48 by LES (Young and Ooi, 2007) and present 3D URANS and SAS calculations

\section{Conclusions}

Three-dimensional calculations were performed to simulate the flow past a cylinder of diameter $D$ at a Reynolds number equal to 3900 using the hybrid turbulence model Scale Adaptive Simulation (SAS). SAS calculations were compared with experiments and Large Eddy Simulations (LES). Predicted overall flow parameters and mean flow velocities showed a very satisfactory agreement with experiments and LES, while the agreement of predicted turbulent stresses was satisfactory. Increasing the vertical dimension of the computation domain from $D$ to $\pi D$ improved only slightly the quality of predictions of the turbulent stresses. The present calculations showed that SAS is an efficient and relatively fast turbulence modeling approach, especially in relevant practical problems, in which the very high accuracy that can be achieved by LES at the expense of large computational times is not required.

\section{Acknowledgement}

The present work was performed within the framework of the Program for Basic Research (PEVE-2010) of the National Technical University of Athens entitled "Computational research of the influence of the vegetation on the flow during floods events".

\section{References}

ANSYS-CFX, Release 14.0, URL: http://www.ANSYS.com.

Ayyappan T. and Vengadesan S. (2008), Three-dimensional simulation of flow past a circular cylinder by nonlinear turbulence model, Numerical Heat Transfer, Part A: Applications, 54(2), 221-234.

Cardell G.S. (1993), Flow past a circular cylinder with a permeable splitter plate, Ph.D. thesis, Graduate Aeronautical Laboratories, California Institute of Technology.

Davidson L. (2006), Evaluation of the SST-SAS model: channel flow, asymmetric diffuser and axi-symmetric hill. European Conference on Computational Fluid Dynamics (ECCOMAS CFD 2006), TU Delft, The Netherlands.

Fairbanks J.D. (1998), Velocity and Turbulence Characteristics in Flows Through Rigid Vegetation, Master of Science in Civil Engineering, Virginia Polytechnic Institute and State University, Blacksburg, Virginia.

Franke J. and Frank W. (2002), Large eddy simulation of the flow past a circular cylinder at Re=3900, Journal of Wind Engineering and Industrial Aerodynamics, 90, 1191-1206.

Kravchenko A.G. and Moin P. (2000), Numerical studies of flow over a circular cylinder at ReD $=3900$, Physics of Fluids, 12(2), 403-417. 
Lopez F. and Garcia M. (2001), Mean flow and turbulence structure of open-channel flow through non-emergent vegetation, Journal of Hydraulic Engineering, 127, 392-402.

Lourenco L.M. and Shih C. (1993), Characteristics of the plane turbulent near wake of a circular cylinder: A particle image velocimetry study, Fluids, 9, 223-253.

Ma X., Karamanos G.S. and Karniadakis G.E. (2000), Dynamic and low-dimensionality of a turbulent near wake, Journal of Fluid Mechanics, 410, 29-65.

Menter F. (1994), Two-Equation Eddy-Viscosity Turbulence Models for Engineering Applications, AIAA Journal, 32(8), 1598-1605.

Menter F. and Egorov Y. (2008), Development and Application of SST-SAS Turbulence Model in the DESIDER Project, Notes on Numerical Fluid Mechanics and Multidisciplinary Design, Springer, 97, 261-270.

Norberg C. (1994), An experimental investigation of the flow around a circular cylinder: influence of aspect ratio, Journal of Fluid Mechanics, 258, 287-316.

Ong L. and Wallace J. (1996), The velocity field of the turbulent very near wake of a circular cylinder, Experiments in Fluids, 20, 441-453.

Prasad A. and Williamson C.H.K. (1996), The instability of the separated shear layer from a bluff body, Physics of Fluids, 8(6), 1347-1349.

Roshko A. (1954), On the development of turbulent wakes from vortex streets, NACA Report 1191.

Stamou A. and Katsiris I. (2006), Verification of a CFD model for indoor airflow and heat transfer, Building and environment, 41(9), 1171-1181.

Stamou A., Papadonikolaki G., Gkesouli A. and Nikoletopoulos A. (2012), Modeling the effect of vegetation on river floodplain hydraulics, Global NEST Journal, 14(3), 371-377.

Widenhorn A., Noll B. and Aigner M. (2008), Numerical Characterization of the Non-Reacting Flow in a Swirled Gasturbine Model Combustor, High Performance Computing in Science and Engineering '07, 431-444.

You J.Y. and Kwon O.J. (2010), Numerical comparisons between URANS and hybrid RANS/LES at high Reynolds number flow using unstructured meshes, International Journal of Aeronautical and Space Sciences, 11, 41-48.

Young M.E. and Ooi A. (2007), Comparative assessment of LES and URANS for flow over a cylinder at Reynolds of 3900, Proceedings of the 16th Australian Fluid Mechanics Conference, Gold Coast, Australia, 1063-1070.

Zhang M., Li C.W. and Shen Y. (2010), A 3D non-linear $k-\varepsilon$ turbulent model for prediction of flow and mass transport in channel with vegetation, Applied Mathematical Modelling, 34, 1021-1031. 